\title{
AN INVESTIGATION OF THE IMPACT OF COMPETITIVE STRATEGY ON EXPORT PERFORMANCE: A LITERATURE REVIEW IN THE PERIOD 1998 - 2018
}

\author{
Katarina Njegić* \\ Faculty of Business Economics and Entrepreneurship \\ Belgrade, Republic of Serbia \\ Nenad Ravić \\ Faculty of Business Economics and Entrepreneurship \\ Belgrade, Republic of Serbia
}

\begin{abstract}
Competitive strategy is considered to be one of the most important determinants of export performance (EP). The last literature review on the link between competitive strategy and EP was provided by Zou and Stan (1998) based on empirical papers published from 1987 until 1997. The aim of this paper is to uncover scientific findings in this field in the period 1998-2008. In order to find empirical studies published in the observed period, a computer and manual bibliographic search was used. In total, 17 empirical studies on the observed topic were revealed. In order to systematize the results of the found studies, a vote-counting technique was used, supplemented with a narrative approach. Several gaps in the literature have been identified and based on them the recommendations for further research in this area are proposed.
\end{abstract}

Key words: competitive strategy, differentiation, low-cost, export performance

JEL classification: $F 23, M 31$

\section{ISPITIVANJE UTICAJA KONKURENTSKE STRATEGIJE NA IZVOZNE PERFORMANE: PREGLED LITERATURE U PERIODU 1998-2018}

Sažetak: Konkurentska strategija se smatra jednom od najvažnijih determinanti izvoznih performansi (IP). Poslednji pregled literature na temu veze između konkurentske strategije i IP ponudili su Zou i Stan (1998) na osnovu empirijskih radova

\footnotetext{
* radosavljevic.k@gmail.com
} 
108 | AN INVESTIGATION OF THE IMPACT OF COMPETITIVE STRATEGY ON EXPORT PERFORMANCE: A LITERATURE REVIEW IN THE PERIOD 1998 - 2018

objavljenih u periodu 1987-1997. Cilj ovog rada je da ustanovi do kojih se naučnih saznanja došlo u ovoj oblasti u periodu od 1998. do 2008. godine. Kako bi se otkrile empirijske studije objavljene u posmatranom periodu, korišćena je kompjuterska $i$ manuelna bibliografska pretraga. Ukupno je otkriveno 17 empirijskih studija na posmatranu temu. Za sistematizaciju rezultata otkrivenih studija korišćena je metoda prebrojavanja glasova, koja je dopunjena narativnim pristupom. Uočeno je nekoliko praznina u literaturi na osnovu kojih se daju preporuke za dalja istraživanja u ovoj oblasti.

Ključne reči: konkurentska strategija, diferencijacija, strategija vođstva u troškovima, izvozne performanse

\section{INTRODUCTION}

Export is the most commonly used form of business involvement in international activities (Morgan, Katsikeas and Vorhies, 2012) and, in today's global world, it is becoming a condition for firms' survival (Acar, 2016). Apart from enabling companies to achieve sustainable growth (Chugan and Singh, 2014), export significantly contributes to the prosperity of the national economy (Ahmed, Julian, Baalbaki and Hadidian, 2004). Therefore, the examination of the factors that affect the firm's success in exporting is of great importance (Njegić and Đokić, 2018). This is evidenced by the large number of studies that are focused on identifying factors that contribute to the increase of the firm's exports (Sousa, Martínez-López and Coelho, 2008; Chen, Sousa and He, 2016). The aim of these studies is to provide managers with guidance for the formulation and implementation of business strategies. The concept that is used for determining the firm's success in exporting is known as export performance $(E P)$ and it is defined as the overall outcome of the firm's activities in export markets (Shoham, 1998).

The empirical examination of the impact of competitive strategy on EP is considered to be one of the most important topics in the field of EP determinants (Chen et al., 2016). Zou and Stan (1998) were the last to present the review of the literature on this topic, which included studies published between 1987 and 1997. The authors found 13 studies that examined the relationship between competitive strategy and some measure of EP. Some studies revealed a positive relationship; however, most of the authors obtained statistically insignificant results in the observed period. Zou and Stan (1998) explained that such results may be the consequence of the lack of a unique way of measuring the implementation of strategy, the reliance on only one EP indicator, and the method used to analyze the data. The second explanation they gave was related to theoretical interpretation. They assumed that the competitive strategy would not necessarily affect EP, but that the results of its 
implementation would depend on how well the firm made the choice of strategic option, and how well that choice fits with the specific circumstances in which the export activity takes place.

The aim of this paper is to systematize the results of empirical research on the impact of the competitive strategy on EP in the period 1998-2018 in order to gain insights into the scientific advances made in this field over the past 20 years. Part of this literature review and some of the found studies were already presented in the doctoral dissertation of the author (Radosavljević, 2017) and they served as the foundation for the formulation of hypotheses in the dissertation. However, due to the importance of this topic, it was decided to expand the literature review and supplement it with the latest empirical studies in order to offer a complete framework of the impact of competitive strategy on EP.

Uncovering the connection between competitive strategy and EP is significant from both a theoretical and a practical point of view. For theorists, establishing a link between competitive strategy and EP contributes to the shaping of the conclusive framework of the determinants of EP. For the researchers in the field of EP, it is useful to have an insight into previously discovered links between the elements of competitive strategy and EP, in order to realize where there is a gap in the literature and the room for further research. In this way, new and original research can be encouraged. From a practical point of view, establishing a link between competitive strategy and EP can be useful for export managers who want to improve the formulation and implementation of the strategy on a scientific basis and thereby to achieve better results in export markets.

The paper is organized into several sections. After the introduction, the theoretical foundations on which the relationship between competitive strategy and EP rests on are presented. In the third part of the paper, a methodological approach that was used for the literature review is presented. The fourth part of the paper summarizes the results obtained by reviewing the empirical studies that were found. The fifth part discusses the theoretical and practical implications of the results, after which a conclusion is drawn and the guidelines for further research are proposed.

\section{THEORETICAL BACKGROUND}

The concept of EP was developed based on the concept of business performance, and today it is considered one of the topics of international management that is most written about but is least understood (Leonidou, Katsikeas and Coudounaris, 2010). One reason for this is that there are different ways of measuring export success. According to the last literature review, over 
110 | AN INVESTIGATION OF THE IMPACT OF COMPETITIVE STRATEGY ON EXPORT PERFORMANCE: A LITERATURE REVIEW IN THE PERIOD 1998 - 2018

50 different EP indicators have been used in previous research (Chen et al., 2016). Although most authors agree that EP is a multidimensional concept, there is no unique perspective on the dimensions of EP. The most common way of examining EP is through financial (economic) and strategic dimension (Papadopulous and Martin, 2010), and sometimes it is observed through the dimensions such as effectiveness, efficiency, and adaptability (Katsikeas, Leonidou and Morgan, 2000).

The next question that concerns researchers is the level at which export performance is analyzed. Two approaches are most common. According to one, EP is observed at the level of the entire export function (indicators of total exports). The second approach is based on observing the export performance of only one export venture in a firm. An export venture refers to a specific product or product line that is exported to one specific export market. The choice between the two approaches causes a debate among the authors, which is understandable since this decision greatly affects the obtained results (Oliveira, Cadogan and Souchon, 2012).

In today's dynamic international environment, exporting firms face severe competition. The way a company chooses to fight competitors in export markets determines a competitive strategy whose aim is to gain and maintain a competitive advantage.

Numerous scientific papers in the field of business strategy started appeared in the literature at the beginning of the 1970s. However, many researchers regard Michael Porter as one of the most influential authors in this field (Eng, 1994). According to Porter, firms can design a competitive strategy to neutralize the competitive forces that rule the market and thus maintain and/or generate above-average profits (Porter, 1979; 1980; 1981; 1985). If a firm wants to achieve above-average results continuously, it must create a sustainable competitive advantage, which requires value creation for its customers (Aaker, 1989). Each business has a number of ways in which it can add value to its consumers, and Porter identifies two main ways. The first is to create added value by increasing the benefits of using the product, and the second is to create additional value by reducing the cost of purchasing the product. According to these ways of creating superior value for the consumer, Porter defines two types of a competitive strategy: 1) differentiation and 2) low-cost (cost leadership) strategy.

A differentiation strategy is based on a firm's effort to offer consumers a product that is different from competing products and thus stand out in the market. In contrast, the basic principle underlying the low-cost strategy is the firm's tendency to continually reduce production costs, which enables the 
company to offer consumers the same value as competitors, but at a lower price, while still generating profits.

According to Porter firms can apply a differentiation strategy or a low-cost strategy in a narrow market segment, thus he defines a third competitive strategy - a focus strategy (Porter, 1980). However, many authors do not consider the focus strategy to be a particular type of competitive strategy, as it does not represent a new way to create competitive advantage. Mintzberg (1988) points out that focus strategy is not a decision on how a firm will gain a competitive advantage, but a decision about the scope of the market. This is the reason why focus strategy is omitted in most research on the topic of Porter's generic strategies (Aulakh, Kotabe and Teegen, 2000).

Both types of competitive strategy have the potential to result in above-average profits, but not every type is equally suitable for every business (Porter, 1985). They differ in many aspects and impose different requirements on resources, skills, control procedures, incentive systems, and management style. Profitability may vary depending on how well the company has aligned its capabilities with the chosen strategy.

An aspect of differentiation that may be problematic is the difficulty of assessing whether additional costs incurred by differentiation will be covered by charging a higher price for the product (Slater and Olson, 2001). If the company is unable to control the additional costs, these can very easily offset the effects of the price premium that has been gained (Miller and Freisen, 1986).

One of the risks of implementing low-cost strategy is the firm's over-focus on cost reduction. If this becomes the dominant philosophy, there is a danger of neglecting important issues in the firm and losing sight of other factors affecting profit (Murray, 1988). In addition, it is argued that if a firm seeks to reduce prices without reducing operating costs, it runs the risk of resource depletion resulting in insolvency, which is particularly dangerous in a market where there is a high level of competition (Woodruff and Zenteno, 2007). A risky aspect of implementing this strategy is the danger of capacity overload, which increases the unit cost of production (Shepherd, 1979).

For the reasons stated above, it is unclear whether and in what situations competitive strategy will lead to better results, and researchers continue to seek empirical confirmation of the impact of competitive strategy on performance, in both domestic and international environments.

\section{METHODOLOGY}

Empirical studies that were chosen had to satisfy several criteria in order to suit the purpose of this paper. In addition to being published in the period 1998- 
112 | AN INVESTIGATION OF THE IMPACT OF COMPETITIVE STRATEGY ON EXPORT PERFORMANCE: A LITERATURE REVIEW IN THE PERIOD 1998 - 2018

2018, it was important that: 1) the selected studies examine one or both types of competitive strategy and establish an empirical link with some of the EP indicators; 2) the firms in the sample use exports as a form of entry into foreign markets; 3) the study clearly states the way the hypotheses were tested, that is, the statistical method that is used, as well as the way the variables were measured in the research.

In order to discover studies with these characteristics, a bibliographic search of the Ebsco database was conducted primarily. In this process, combinations of the following keywords were used: competitive strategy, general strategy, generic strategy, business strategy, differentiation, cost leadership, low-cost strategy, export performance, international performance, and export success. Search engines sometimes cannot identify studies where target relationships are studied as a secondary topic. In order to identify studies that examine the observed relationships as part of a broader research problem, a manual search of the list of references in the discovered studies was also conducted.

To review the literature vote-counting technique supplemented with a narrative approach was used. Vote-counting technique involves counting the revealed statistically positive, negative or statistically insignificant relationships between the observed variables reported by the authors (Hedges and Olkin, 1980). The narrative approach served to further explain revealed relationships, as well as to explain the way in which the variables were measured in the revealed studies. The combination of these two methods is considered to be suitable when the operationalization of variables in studies differs, as it is the case in EP research (Sousa et al., 2008). The advantages of the vote-counting technique are clarity and transparency, and it allows researchers to draw their own conclusions (Zou and Stan, 1998). This method is also used by renowned authors in this field (Theodosiou and Leonidou, 2003; Sousa et al., 2008).

A total of 17 studies on the observed topic that were published in the period 1998-2018 were discovered. Apart from the studies that examine the impact of competitive strategy on EP, bibliographic search also revealed studies that examine the impact of competitive advantage (differentiation and/or low-cost advantage) on EP (five out of 17 studies). Since competitive advantage is a direct consequence of the adoption of competitive strategy, there is a theoretical basis for the inclusion of these studies as well. From a practical point of view, the only difference between these two types of studies is that in studies examining the implementation of a competitive strategy respondents evaluate the extent to which their firm adopts certain elements of the strategy, while in the studies that examine competitive advantage respondents are asked to evaluate similar elements by comparing their firm with its major competitors in 
export markets. The characteristics of all the found studies are summarized in Table 1 . The studies were sorted chronologically. 
114 | AN INVESTIGATION OF THE IMPACT OF COMPETITIVE STRATEGY ON EXPORT PERFORMANCE: A LITERATURE REVIEW IN THE PERIOD 1998 - 2018

Table 1

Characteristics of the reviewed studies (I part)

\begin{tabular}{|c|c|c|c|c|c|c|c|c|c|}
\hline Author(s) & $\begin{array}{l}\text { Country } \\
\text { of study }\end{array}$ & $\begin{array}{l}\text { Sample } \\
\text { size and } \\
\text { response } \\
\text { rate }(\%)\end{array}$ & $\begin{array}{l}\text { Industrial } \\
\text { sector }\end{array}$ & $\begin{array}{l}\text { Firm } \\
\text { size in } \\
\text { the } \\
\text { sample }\end{array}$ & $\begin{array}{l}\text { Data } \\
\text { collection } \\
\text { method }\end{array}$ & $\begin{array}{l}\text { Key } \\
\text { informant }\end{array}$ & $\begin{array}{l}\text { EP measure } \\
\text { used }\end{array}$ & $\begin{array}{l}\text { Unit of } \\
\text { analysis }\end{array}$ & $\begin{array}{l}\text { Statistical } \\
\text { method } \\
\text { used }\end{array}$ \\
\hline $\begin{array}{l}\text { Aulakh et } \\
\text { al. (2000) }\end{array}$ & $\begin{array}{l}\text { Brazil } \\
\text { Chile } \\
\text { Mexico }\end{array}$ & $\begin{array}{l}80(27.2) \\
80(44.4) \\
36(100)\end{array}$ & $\begin{array}{l}\text { Manufacturing } \\
\text { and service } \\
\text { firms from } \\
\text { different } \\
\text { industries }\end{array}$ & $\begin{array}{l}\text { All } \\
\text { sizes }\end{array}$ & $\begin{array}{l}\text { Mail survey } \\
\text { and interview }\end{array}$ & $\begin{array}{l}\text { Upper } \\
\text { managers }\end{array}$ & $\begin{array}{l}\text {-composite } \\
\text { measure of } \\
\text { EP }\end{array}$ & Firm-level & $\begin{array}{l}\text { Hierarchical } \\
\text { regression } \\
\text { analysis }\end{array}$ \\
\hline $\begin{array}{l}\text { Baldauf } e t \\
\text { al. }(2000)\end{array}$ & Austria & $184(53)$ & $\begin{array}{l}\text { Manufacturing } \\
\text { firms from } \\
\text { different } \\
\text { industries } \\
\text { (industrial and } \\
\text { consumer } \\
\text { goods) }\end{array}$ & $\begin{array}{l}\text { All } \\
\text { sizes }\end{array}$ & Mail survey & $\begin{array}{l}\text { CEO or } \\
\text { vice- } \\
\text { president } \\
\text { of } \\
\text { marketing } \\
\text { or } \\
\text { international } \\
\text { operations }\end{array}$ & $\begin{array}{l}\text {-export } \\
\text { effectiveness, } \\
\text { export } \\
\text { intensity, } \\
\text { export sales } \\
\text { (revenue) }\end{array}$ & Firm-level & $\begin{array}{l}\text { Regression } \\
\text { analysis }\end{array}$ \\
\hline $\begin{array}{l}\text { Ling-Yee, \& } \\
\text { Ogunmokun } \\
(2001)\end{array}$ & China & $\begin{array}{l}111 \\
(39.6)\end{array}$ & $\begin{array}{l}\text { Manufacturing } \\
\text { firms from } \\
\text { different } \\
\text { industries }\end{array}$ & $\begin{array}{l}\text { All } \\
\text { sizes }\end{array}$ & Mail survey & $\begin{array}{l}\text { Export } \\
\text { managers }\end{array}$ & $\begin{array}{l}\text {-financial EP } \\
\text {-achievement } \\
\text { of export } \\
\text { strategic } \\
\text { goals }\end{array}$ & $\begin{array}{l}\text { Export } \\
\text { venture }\end{array}$ & $\begin{array}{l}\text { Regression } \\
\text { analysis }\end{array}$ \\
\hline $\begin{array}{l}\text { Brouthers, } \\
\text { \& Xu (2002) }\end{array}$ & China & $\begin{array}{l}186 \\
(47.3)\end{array}$ & $\begin{array}{l}\text { Manufacturing } \\
\text { firms from } \\
\text { different } \\
\text { industries }\end{array}$ & $\begin{array}{l}\text { All } \\
\text { sizes }\end{array}$ & Interview & $\begin{array}{l}\text { Export } \\
\text { managers }\end{array}$ & $\begin{array}{l}\text {-satisfaction } \\
\text { with EP }\end{array}$ & Firm-level & $\begin{array}{l}\text { Regression } \\
\text { analysis }\end{array}$ \\
\hline
\end{tabular}


Table 1 (II part)

\begin{tabular}{|c|c|c|c|c|c|c|c|c|c|}
\hline Author(s) & $\begin{array}{l}\text { Country } \\
\text { of study }\end{array}$ & $\begin{array}{l}\text { Sample } \\
\text { size and } \\
\text { response } \\
\text { rate }(\%)\end{array}$ & $\begin{array}{l}\text { Industrial } \\
\text { sector }\end{array}$ & $\begin{array}{l}\text { Firm } \\
\text { size in } \\
\text { the } \\
\text { sample }\end{array}$ & $\begin{array}{l}\text { Data } \\
\text { collection } \\
\text { method }\end{array}$ & $\begin{array}{l}\text { Key } \\
\text { informant }\end{array}$ & $\begin{array}{l}\text { EP measure } \\
\text { used }\end{array}$ & $\begin{array}{l}\text { Unit of } \\
\text { analysis }\end{array}$ & $\begin{array}{l}\text { Statistical } \\
\text { method } \\
\text { used }\end{array}$ \\
\hline $\begin{array}{l}\text { Zou et al. } \\
(2003)\end{array}$ & China & $\begin{array}{l}176 \\
(75.0)\end{array}$ & $\begin{array}{l}\text { Manufacturing } \\
\text { firms from } \\
\text { different } \\
\text { industries }\end{array}$ & $\begin{array}{l}\text { All } \\
\text { sizes }\end{array}$ & Mail survey & $\begin{array}{l}\text { export } \\
\text { marketing } \\
\text { manager }\end{array}$ & $\begin{array}{l}\text {-financial } \\
\text { EP }\end{array}$ & $\begin{array}{l}\text { Export } \\
\text { venture }\end{array}$ & $\begin{array}{l}\text { Structural } \\
\text { equation } \\
\text { modeling } \\
(\mathrm{SEM})\end{array}$ \\
\hline Man (2009) & Malezia & $121(/)$ & $\begin{array}{l}\text { Manufacturing } \\
\text { firms from } \\
\text { different } \\
\text { industries }\end{array}$ & $\begin{array}{l}\text { Small } \\
\text { and } \\
\text { medium }\end{array}$ & Mail survey & $\begin{array}{l}\text { Export } \\
\text { managers }\end{array}$ & $\begin{array}{l}\text {-export sales } \\
\text { (revenue) }\end{array}$ & Firm-level & $\begin{array}{l}\text { Regression } \\
\text { analysis }\end{array}$ \\
\hline $\begin{array}{l}\text { Salavou, } \\
\text { \& Halikias } \\
(2009)\end{array}$ & Greece & $82(25.3)$ & $\begin{array}{l}\text { Manufacturing } \\
\text { firms from } \\
\text { different } \\
\text { industries }\end{array}$ & $\begin{array}{l}\text { All } \\
\text { sizes }\end{array}$ & Mail survey & $\begin{array}{l}\text { general and } \\
\text { export } \\
\text { managers }\end{array}$ & $\begin{array}{l}\text {-export } \\
\text { profitability }\end{array}$ & Firm-level & $\begin{array}{l}\text { K-means } \\
\text { cluster } \\
\text { analysis; } \\
\text { one-way } \\
\text { ANOVAs; } \\
\text { MDA }\end{array}$ \\
\hline $\begin{array}{l}\text { Boehe, \& } \\
\text { Cruz (2010) }\end{array}$ & Brazil & $252(7.5)$ & $\begin{array}{l}\text { Manufacturing } \\
\text { firms from } \\
\text { different } \\
\text { industries }\end{array}$ & $\begin{array}{l}\text { All } \\
\text { sizes }\end{array}$ & E-mail survey & $\begin{array}{l}\text { main } \\
\text { decision- } \\
\text { maker } \\
\text { for the } \\
\text { firm's } \\
\text { export } \\
\text { business }\end{array}$ & $\begin{array}{l}\text {-composite } \\
\text { EP }\end{array}$ & Firm-level & SEM \\
\hline $\begin{array}{l}\text { Hughes et } \\
\text { al. (2010) }\end{array}$ & Mexico & $\begin{array}{l}260 \\
(19.8)\end{array}$ & $\begin{array}{l}\text { High- } \\
\text { technology } \\
\text { firms }\end{array}$ & $\begin{array}{l}\text { All } \\
\text { sizes }\end{array}$ & $\begin{array}{l}\text { Telephone } \\
\text { interview }\end{array}$ & $\begin{array}{l}\text { Export } \\
\text { managers }\end{array}$ & $\begin{array}{l}\text {-composite } \\
\text { EP }\end{array}$ & $\begin{array}{l}\text { Export } \\
\text { venture }\end{array}$ & SEM \\
\hline
\end{tabular}


116 | AN INVESTIGATION OF THE IMPACT OF COMPETITIVE STRATEGY ON EXPORT PERFORMANCE: A LITERATURE REVIEW IN THE PERIOD 1998 - 2018

Table 1 (III part)

\begin{tabular}{|c|c|c|c|c|c|c|c|c|c|}
\hline Author(s) & $\begin{array}{l}\text { Country } \\
\text { of study }\end{array}$ & $\begin{array}{l}\text { Sample } \\
\text { size and } \\
\text { response } \\
\text { rate }(\%)\end{array}$ & $\begin{array}{l}\text { Industrial } \\
\text { sector }\end{array}$ & $\begin{array}{l}\text { Firm } \\
\text { size in } \\
\text { the } \\
\text { sample }\end{array}$ & $\begin{array}{l}\text { Data } \\
\text { collection } \\
\text { method }\end{array}$ & $\begin{array}{l}\text { Key } \\
\text { informant }\end{array}$ & $\begin{array}{l}\text { EP } \\
\text { measure } \\
\text { used }\end{array}$ & $\begin{array}{l}\text { Unit of } \\
\text { analysis }\end{array}$ & $\begin{array}{l}\text { Statistical } \\
\text { method } \\
\text { used }\end{array}$ \\
\hline $\begin{array}{l}\text { Leonidou } e t \\
\text { al. (2011) }\end{array}$ & $\begin{array}{l}\text { United } \\
\text { Kingdom }\end{array}$ & $\begin{array}{l}223 \\
(51.8)\end{array}$ & $\begin{array}{l}\text { Manufacturing } \\
\text { firms from } \\
\text { different } \\
\text { industries }\end{array}$ & $\begin{array}{l}\text { All } \\
\text { sizes }\end{array}$ & $\begin{array}{l}\text { Mail and e- } \\
\text { mail survey }\end{array}$ & $\begin{array}{l}\text { Export } \\
\text { managers }\end{array}$ & $\begin{array}{l}\text {-financial } \\
\text { EP } \\
\text {-export } \\
\text { market } \\
\text { performance }\end{array}$ & Firm-level & SEM \\
\hline $\begin{array}{l}\text { Murray et } \\
\text { al. (2011) }\end{array}$ & China & $\begin{array}{l}491 \\
(37.0)\end{array}$ & $\begin{array}{l}\text { Manufacturing } \\
\text { firms from } \\
\text { different } \\
\text { industries }\end{array}$ & $\begin{array}{l}\text { All } \\
\text { sizes }\end{array}$ & Interview & $\begin{array}{l}\text { Export } \\
\text { managers }\end{array}$ & $\begin{array}{l}\text {-financial } \\
\text { EP } \\
\text {-strategic } \\
\text { EP } \\
\text {-product } \\
\text { performance }\end{array}$ & Firm-level & SEM \\
\hline $\begin{array}{l}\text { Okpara } \\
(2012)\end{array}$ & Nigeria & $\begin{array}{l}178 \\
(69.0)\end{array}$ & $\begin{array}{l}\text { Manufacturing } \\
\text { firms (food } \\
\text { and beverage, } \\
\text { agriculture, } \\
\text { textile, } \\
\text { clothing, } \\
\text { footwear, } \\
\text { leather, } \\
\text { chemistry) }\end{array}$ & $\begin{array}{l}\text { Small } \\
\text { and } \\
\text { medium }\end{array}$ & Interview & managers & $\begin{array}{l}\text {-export sales } \\
\text { growth } \\
\text {-export } \\
\text { profitability } \\
\text {-overall EP }\end{array}$ & Firm-level & $\begin{array}{l}\text { Cluster } \\
\text { analysis }\end{array}$ \\
\hline $\begin{array}{l}\text { Kumlu } \\
\text { (2014) }\end{array}$ & Turkey & $\begin{array}{l}271 \\
(22.0)\end{array}$ & $\begin{array}{l}\text { Manufacturing } \\
\text { firms (metal, } \\
\text { chemistry, } \\
\text { textile and } \\
\text { clothing, } \\
\text { furniture) }\end{array}$ & $\begin{array}{l}\text { Small } \\
\text { and } \\
\text { medium }\end{array}$ & Mail survey & $\begin{array}{l}\text { Export } \\
\text { managers }\end{array}$ & $\begin{array}{l}\text {-composite } \\
\text { EP }\end{array}$ & Firm-level & $\begin{array}{l}\text { Regression } \\
\text { analysis }\end{array}$ \\
\hline
\end{tabular}


Table 1 (IV part)

\begin{tabular}{|c|c|c|c|c|c|c|c|c|c|}
\hline Author(s) & $\begin{array}{l}\text { Country } \\
\text { of study }\end{array}$ & $\begin{array}{l}\text { Sample } \\
\text { size and } \\
\text { response } \\
\text { rate }(\%)\end{array}$ & $\begin{array}{l}\text { Industrial } \\
\text { sector }\end{array}$ & $\begin{array}{l}\text { Firm } \\
\text { size in } \\
\text { the } \\
\text { sample }\end{array}$ & $\begin{array}{l}\text { Data } \\
\text { collection } \\
\text { method }\end{array}$ & $\begin{array}{l}\text { Key } \\
\text { informant }\end{array}$ & $\begin{array}{l}\text { EP } \\
\text { measure } \\
\text { used }\end{array}$ & $\begin{array}{l}\text { Unit of } \\
\text { analysis }\end{array}$ & $\begin{array}{l}\text { Statistical } \\
\text { method } \\
\text { used }\end{array}$ \\
\hline $\begin{array}{l}\text { Leonidou } \text { et } \\
\text { al. (2015) }\end{array}$ & Greece & $\begin{array}{l}233 \\
(44.5)\end{array}$ & $\begin{array}{l}\text { Manufacturing } \\
\text { firms from } \\
\text { different } \\
\text { industries }\end{array}$ & $\begin{array}{l}\text { Medium } \\
\text { and } \\
\text { large }\end{array}$ & Mail survey & $\begin{array}{l}\text { Export } \\
\text { managers }\end{array}$ & $\begin{array}{l}\text {-financial } \\
\text { EP } \\
\text {-export } \\
\text { market } \\
\text { performance }\end{array}$ & Firm-level & SEM \\
\hline $\begin{array}{l}\text { Erdil, } \\
\text { Özdemir } \\
\text { (2016) }\end{array}$ & Turkey & $118(/)$ & $\begin{array}{l}\text { Manufacturing } \\
\text { firms from } \\
\text { textile and } \\
\text { clothing } \\
\text { industry }\end{array}$ & $\begin{array}{l}\text { All } \\
\text { sizes }\end{array}$ & $\begin{array}{l}\text { Online survey } \\
\text { and interview }\end{array}$ & $\begin{array}{l}\text { Top } \\
\text { managers }\end{array}$ & $\begin{array}{l}\text {-financial } \\
\text { EP }\end{array}$ & Firm-level & $\begin{array}{l}\text { Regression } \\
\text { analysis }\end{array}$ \\
\hline $\begin{array}{l}\text { Martin et al. } \\
\text { (2017) }\end{array}$ & Mexico & $\begin{array}{l}260 \\
(19.8)\end{array}$ & $\begin{array}{l}\text { High-tech } \\
\text { born global } \\
\text { Firms }\end{array}$ & $\begin{array}{l}\text { Small } \\
\text { and } \\
\text { medium }\end{array}$ & $\begin{array}{l}\text { Telephone } \\
\text { interview }\end{array}$ & $\begin{array}{l}\text { Executive } \\
\text { managers }\end{array}$ & $\begin{array}{l}\text {-composite } \\
\text { EP }\end{array}$ & $\begin{array}{l}\text { Export } \\
\text { venture }\end{array}$ & $\begin{array}{l}\text { SEM } \\
\text { (elliptical } \\
\text { reweighted } \\
\text { least } \\
\text { squares } \\
\text { estimation) }\end{array}$ \\
\hline $\begin{array}{l}\text { Rua et al. } \\
\text { (2018) }\end{array}$ & Portugal & $247(25)$ & $\begin{array}{l}\text { Textile } \\
\text { industry }\end{array}$ & $\begin{array}{l}\text { Small } \\
\text { and } \\
\text { medium }\end{array}$ & Survey & $\begin{array}{l}\text { Not } \\
\text { specified }\end{array}$ & $\begin{array}{l}\text {-composite } \\
\text { EP }\end{array}$ & Firm-level & PLS-SEM \\
\hline
\end{tabular}

Note. Authors' research. 
The countries where the studies were conducted are listed in the second column of Table 1. It can be noted that studies from developed and emerging countries are prevalent. The sample size in the found studies ranged from 82 to 491 firms, while the response rate ranged from 7,5\% to $75 \%$ (third column). In most of the studies, authors examine manufacturing firms from different industries (fourth column). Samples that cover firms of all sizes are also dominant (fifth column). This approach allows researchers to subsequently control factors such as company size and industry sector. The data were collected mainly by mail survey (sixth column) and the key respondents were mostly export managers, although there are studies that survey general managers (seventh column). Important aspects of the relationship between competitive strategy and EP are the way EP is measured, the level at which EP is evaluated (unit of analysis), and the statistical method used to test the hypotheses. This information is presented in the last three columns of Table 1, and a detailed description of how the authors measured EP is given in the Appendix. Most authors examined EP at the firm level (13 studies), while only four studies examined EP at the export venture level. The most common statistical methods used were structural equation modeling (SEM) and regression analysis, with only two studies using cluster analysis. It can be noted that regression analysis as a method was present at the beginning of the observed period. In more recent research, it has been replaced by more sophisticated methods such as SEM, which allows researchers to test causal relationships between variables.

\section{RESULTS AND DISCUSSION}

As can be seen from the previous table (Table 1), numerous indicators of EP were used. In the observed period, various financial indicators of EP were frequently used (in 10 studies). The authors often formulated their own composite measures of EP, consisting of different indicators and covering multiple dimensions of EP (in eight studies found). In the studies found, the authors rarely include the strategic dimension of EP (in only two studies), although it is considered to be an important aspect of EP, especially in strategy research. Table 2 summarizes the results of the literature review. Relationships between different types of competitive strategy (competitive advantage) and different EP indicators that authors discovered during the observed period are counted and presented in the table. 
Table 2

Discovered relationships between competitive strategy (advantage) and EP (I part)

\begin{tabular}{|c|c|c|c|c|c|c|c|c|c|c|c|c|}
\hline \multirow{2}{*}{$\begin{array}{l}\text { Competitive } \\
\text { strategy/ } \\
\text { competitive } \\
\text { advantage }\end{array}$} & \multicolumn{12}{|l|}{ EP measures } \\
\hline & 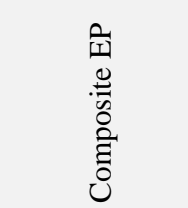 & 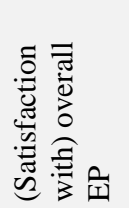 & 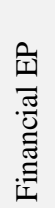 & 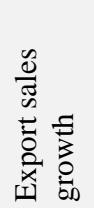 & 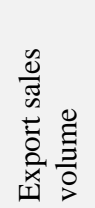 & 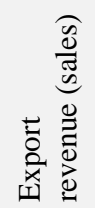 & 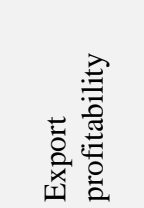 & 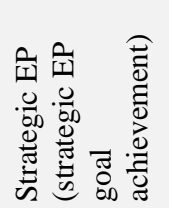 & 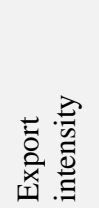 & 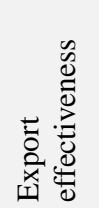 & 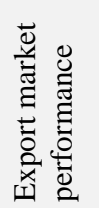 & 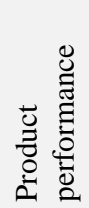 \\
\hline $\begin{array}{l}\text { Differentiation } \\
\text { strategy }\end{array}$ & $\begin{array}{l}2(+) \\
\text { Aulakh et al. (2000), } \\
\text { Kumlu (2014) } \\
1(0) \\
\text { Rua et al. (2018) }\end{array}$ & $\begin{array}{l}1(+) \\
\text { Okpara } \\
(2012)\end{array}$ & & $\begin{array}{l}1 \\
(+) \\
\text { Okpara } \\
(2012)\end{array}$ & $\begin{array}{l}1(-) \\
\text { Baldauf } \\
\text { et al. } \\
(2000)\end{array}$ & $\begin{array}{l}1(+) \\
\text { Man } \\
(2009)\end{array}$ & $\begin{array}{l}2(+): \\
\text { Salavou, \& } \\
\text { Halikias } \\
\text { (2009); } \\
\text { Okpara (2012) }\end{array}$ & & $\begin{array}{l}1(-) \\
\text { Baldauf } \\
\text { et al. } \\
(2000)\end{array}$ & $\begin{array}{l}1(+) \\
\text { Baldauf } \\
\text { et al. } \\
(2000)\end{array}$ & & \\
\hline $\begin{array}{l}\text { Brand } \\
\text { differentiation }\end{array}$ & & $\begin{array}{l}1(+) \\
\text { Brouthers, } \\
\text { Xu (2002) }\end{array}$ & & & & & & & & & & \\
\hline $\begin{array}{l}\text { Differentiation } \\
\text { based on quality }\end{array}$ & $\begin{array}{l}\text { 1(0) } \\
\text { Boehe, Cruz (2010) }\end{array}$ & & & & & & & & & & & \\
\hline $\begin{array}{l}\text { Differentiation } \\
\text { based on } \\
\text { innovation }\end{array}$ & $\begin{array}{l}1(+) \\
\text { Boehe, Cruz (2010) }\end{array}$ & & & & & & & & & & & \\
\hline $\begin{array}{l}\text { Differentiation } \\
\text { focus strategy }\end{array}$ & & $\begin{array}{l}1(+) \\
\text { Okpara } \\
(2012)\end{array}$ & & $\begin{array}{l}1(+) \\
\text { Okpara } \\
(2012)\end{array}$ & & & $\begin{array}{l}2(+) \\
\text { Okpara } \\
(2012) ; \\
\text { Salavou, } \\
\text { Halikias } \\
(2009)\end{array}$ & & & & & \\
\hline
\end{tabular}


120 | AN INVESTIGATION OF THE IMPACT OF COMPETITIVE STRATEGY ON EXPORT PERFORMANCE: A LITERATURE REVIEW IN THE PERIOD 1998 - 2018

Table 2 (II part)

\begin{tabular}{|c|c|c|c|c|c|c|c|c|c|c|c|c|}
\hline Competitive & EP measures & & & & & & & & & & & \\
\hline $\begin{array}{l}\text { strategy/ } \\
\text { competitive } \\
\text { advantage }\end{array}$ & 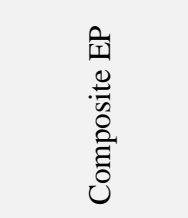 & 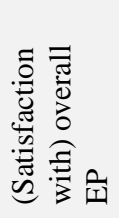 & 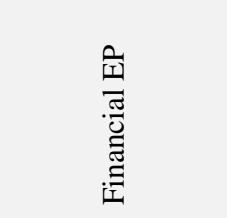 & 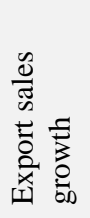 & 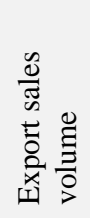 & 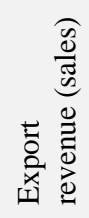 & 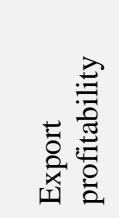 & 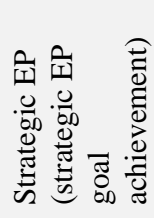 & 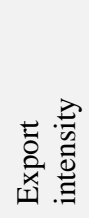 & 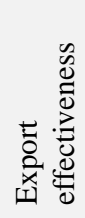 & 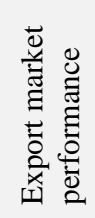 & 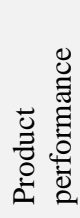 \\
\hline $\begin{array}{l}\text { Low-cost } \\
\text { strategy }\end{array}$ & $\begin{array}{l}3(+) \text { Aulakh et al. } \\
(2000) ; \text { Kumlu } \\
\text { (2014); Rua et al. } \\
(2018)\end{array}$ & $\begin{array}{l}1(+) \\
\text { Okpara } \\
(2012) \\
1(-) \\
\text { Brouthers, } \\
\text { Xu (2002) }\end{array}$ & & $\begin{array}{l}1(+) \\
\text { Okpara } \\
(2012)\end{array}$ & $\begin{array}{l}1(0) \\
\text { Baldauf } \\
\text { et al. } \\
(2000)\end{array}$ & $\begin{array}{l}1(0) \\
\text { Man } \\
(2009)\end{array}$ & $\begin{array}{l}2(+) \\
\text { Okpara } \\
(2012) ; \\
\text { Salavou, } \\
\text { Halikias } \\
(2009)\end{array}$ & & $\begin{array}{l}1(0) \\
\text { Baldauf } \\
\text { et al. } \\
(2000)\end{array}$ & $\begin{array}{l}1(-) \\
\text { Baldauf } \\
\text { et al. } \\
(2000)\end{array}$ & & \\
\hline $\begin{array}{l}\text { Competitive } \\
\text { strategy }\end{array}$ & $\begin{array}{l}1(0) \\
\text { Martin et al. (2017) }\end{array}$ & & & & & & & & & & & \\
\hline $\begin{array}{l}\text { Differentiation } \\
\text { competitive } \\
\text { advantage }\end{array}$ & $\begin{array}{l}1(+) \\
\text { Hughes et al. (2010) }\end{array}$ & & $\begin{array}{l}\text { 3(+) } \\
\text { Murray et al. (2011); } \\
\text { Leonidou et al. (2015); } \\
\text { Erdil, Ozemir (2016) } \\
2(0) \\
\text { Ling-Yee \& } \\
\text { Ogunmokun (2001); } \\
\text { Leonidou et al. (2011) }\end{array}$ & & & & & $\begin{array}{l}2(+) \\
\text { Ling-Yee \& } \\
\text { Ogunmokun } \\
(2001) ; \\
\text { Murray et al. } \\
(2011)\end{array}$ & & & $\begin{array}{l}2(+) \\
\text { Leonidou } \\
\text { et al. } \\
(2011) \text {; } \\
\text { Leonidou } \\
\text { et al. } \\
(2015)\end{array}$ & $\begin{array}{l}1(+) \\
\text { Murray } \\
\text { et al. } \\
(2011)\end{array}$ \\
\hline $\begin{array}{l}\text { Low-cost } \\
\text { competitive } \\
\text { advantage }\end{array}$ & $\begin{array}{l}1(+) \\
\text { Hughes et al. (2010) }\end{array}$ & & $\begin{array}{l}\text { 4(+) } \\
\text { Murray et al. (2011); } \\
\text { Leonidou et al. (2011); } \\
\text { Ling-Yee \& } \\
\text { Ogunmokun (2001); Zou } \\
\text { et al. (2003) } \\
1(0) \\
\text { Leonidou et al. (2015) }\end{array}$ & & & & & $\begin{array}{l}1(+) \\
\text { Murray et al. } \\
(2011) \\
1(0) \\
\text { Ling-Yee \& } \\
\text { Ogunmokun } \\
(2001)\end{array}$ & & & $\begin{array}{l}1(+) \\
\text { Leonidou } \\
\text { et al. } \\
(2011) \\
1(0) \\
\text { Leonidou } \\
\text { et al. } \\
(2015)\end{array}$ & $\begin{array}{l}1(0) \\
\text { Murray } \\
\text { et al. } \\
(2011)\end{array}$ \\
\hline
\end{tabular}

Note. Authors' research. 
Based on the results reported by the authors over the observed period, it is evident that differentiation strategy has a positive impact on the financial dimension of EP. In studies that have examined the differentiation strategy and export sales (revenue or growth), the assumption of a positive relationship between the observed variables was confirmed in most of the cases. In one study it is hypothesized that differentiation is going to have a negative impact on export sales volume (Baldauf, Cravens and Wagner, 2000). The authors explain this by the fact that differentiation strategy involves serving a smaller number of consumers who are willing to pay a price premium, that is, selling fewer products at a higher price, which will provide the necessary profit. They confirm their hypothesis and obtain a statistically significant negative relationship between differentiation strategy and export sales volume and export intensity. The results of the conducted review showed that differentiation strategy positively affects export profitability (Salavou and Halikias, 2009; Okpara, 2012) and export effectiveness (Baldauf et al., 2000). It was also found that differentiation leads to better overall EP (Okpara, 2012) and that it has a positive effect on composite EP (Aulakh et al., 2000; Kumlu, 2014), although, there are authors who report on statistically insignificant results when testing this relationship (Rua, França and Fernández Ortiz, 2018). There is little evidence of the impact of specific types of differentiation strategy on EP. The results revealed that brand differentiation strategy has a positive effect on overall EP (Brouthers and $\mathrm{Xu}, 2002)$, that differentiation based on innovation positively affects the composite outcome of export activities (Boehe and Cruz 2010), and that quality-based differentiation does not affect composite EP (Boehe and Cruz, 2010).

The examination of the impact of differentiation on competitive advantage showed positive results in most of the cases. The authors report on the positive impact of differentiation-based competitive advantage on composite EP (Hughes, Martin, Morgan and Robson, 2010), strategic dimension of EP (LingYee and Ogunmokun, 2001; Murray, Gao and Kotabe, 2011), as well as on export market performance (Leonidou et al., 2011; Leonidou et al., 2015), product performance (Murray et al., 2011), and financial dimension of EP (Murray et al., 2011; Leonidou et al., 2015; Erdil and Ozemir, 2016). Few authors examined the impact of a differentiation focus strategy on EP. The conclusion of these studies is that this strategy contributes to export sales growth and overall EP (Okpara, 2012) and that it increases export profitability (Salavou and Halikias, 2009; Okpara, 2012).

The literature review that was conducted led to some interesting insights into the link between low-cost strategy and EP. The implementation of a low-cost strategy was shown to increase export sales growth (Okpara, 2012) and export profitability (Okpara, 2012; Salavou and Halikias, 2009). However, it is unclear 
122 | AN INVESTIGATION OF THE IMPACT OF COMPETITIVE STRATEGY ON EXPORT PERFORMANCE: A LITERATURE REVIEW IN THE PERIOD 1998 - 2018

how this strategy will affect export sales volume, export sales revenue, and export intensity since only individual studies test these relationships and they reveal statistically insignificant results (Baldauf et al., 2000; Man, 2009). It is also questionable how low-cost strategy will affect the overall EP, given that the authors of one study report on a positive relationship (Okpara, 2012) while another group of authors report on a negative relationship between low-cost strategy and this EP indicator (Brouthers and $\mathrm{Xu}, 2002)$. Low-cost strategy was shown to have a negative effect on export effectiveness (Baldauf et al., 2000). This finding can be explained by the high level of investment involved in the implementation of this strategy as well as by the problem of maintaining lowcost benefits in the long run (Leonidou et al., 2015). Authors who used composite measures of EP report on a positive impact of low-cost strategy on EP (Aulakh et al., 2000; Kumlu, 2014; Rua et al., 2018).

Testing of the relationship between low-cost competitive advantage and EP generally yielded consistent results. This type of competitive advantage in the export market was shown to lead to better financial EP, as evidenced by four studies (Murray et al., 2011; Leonidou et al., 2011; Ling-Yee and Ogunmokun, 2001; Zou, Fang and Zhao, 2003). Only one study that tested this relationship reported on statistically insignificant results (Leonidou et al., 2015). Low-cost advantage was also shown to have a positive effect on composite EP (Hughes et al., 2010), but it is still unclear how it will affect product performance (Murray et al., 2011).

In one study found, the authors measure the level of implementation of a competitive strategy without separating the differentiation strategy and low-cost strategy (Martin, Javalgi and Cavusgil, 2017). This type of operationalization is not common. The authors of this study report on a statistically insignificant relationship between competitive strategy and composite EP. Given that this research is unique, this relationship needs further examination.

\section{DIRECTIONS FOR FUTURE RESEARCH}

Based on the results of the conducted review, it can be concluded that it is not sufficiently examined how certain types of competitive strategy will affect EP. The systematization of the results is further complicated by the large number of EP indicators and the different ways in which authors measure EP in their research. When interpreting the results, the way in which the sample was formed should be taken into account as well as the external environment in which the research was conducted. Some authors emphasize that the size of the firm, the level of development of the country of origin, and the industry sector determine the type of competitive strategy that the firm will implement. For example, industrial products are thought to have less potential for differentiation (Aulakh et al., 2000). Murrey and her colleagues state that firms exporting from 
developing countries generally adopt low-cost strategy due to the low labor costs and low prices of raw materials (Murray et al., 2011). It is also stated that small businesses find it difficult to differentiate themselves in an international market where the competition is intense (Murray et al., 2011; Rua et al., 2018).

Although the impact of competitive strategy on EP is a significant topic in international business and strategic management, relatively few papers were published in the observed period. This indicates that there is room for further research in this field. In particular, studies from developing countries are scarce. The review that was conducted revealed some other gaps in the literature. Most authors create samples that include manufacturing firms from a variety of industry sectors. Therefore, it would be interesting to gain insights from individual industries. It was also found that authors rarely control for the size of the company in their research and they form samples from firms of all sizes. Therefore, it would be interesting to uncover how specific types of competitive strategy will affect EP of firms different in sizes. The systematization of the results and the drawing of the final conclusions about the impact of competitive strategy on EP is difficult not only because of the large number of EP indicators but also because of the fact that the authors measure the level of implementation of the competitive strategy differently. Therefore, future research could go towards formulating a unique measure of the competitive strategy for export markets. In the studies found, the strategic dimension of EP received little attention. Therefore, future research could include some of the strategic EP indicators such as the level of achievement of the firm's export strategic goals. For authors who wish to examine this important topic, it is recommended to use more complex statistical methods, such as structural equation modeling, since regression analysis is simple and does not provide insight into the causal relationships between the tested variables.

\section{CONCLUSIONS}

The relationship between competitive strategy and EP is a very important topic in the research of EP determinants. However, no literature review that summarizes the results of previous research on this issue has been done in the last 20 years. In order to fill this gap in the literature, a review of empirical studies published in the period 1998-2018 was conducted.

The review provided significant insights into the relationship between competitive strategy and EP. In the period under review, based on theoretical postulates set by Porter $(1979 ; 1980 ; 1985)$, the authors generally assumed a positive relationship between competitive strategy and EP. However, as a large number of different indicators are used in EP research and there is no established unique way of measuring EP, the authors report on different results. Different results may be present due to the different environments (countries) in 
which the research was conducted. The results are also influenced by the way the sample is made, especially the size of the firms covered by the study. This should be borne in mind when designing future research frameworks.

Although it provides interesting insights into the link between the competitive strategy and the EP, some limitations of the presented study should be kept in mind. This primarily relates to the disadvantage of electronic bibliographical search by keyword. It is possible that some published study was unintentionally omitted. Another limitation of the presented review is that it includes only studies that are published in English.

\section{REFERENCES}

Aaker, D. A. (1989). Managing assets and skills: The key to a sustainable competitive advantage. California management review, 31(2), 91-106.

Acar, F. P. (2016). The effects of top management team composition on SME export performance: an upper echelons perspective. Central European Journal of Operations Research, 24(4), 833-852.

Ahmed, Z. U., Julian, C. C., Baalbaki, I., \& Hadidian, T. V. (2004). Export barriers and firm internationalisation: a study of Lebanese entrepreneurs. Journal of Management and World Business Research, 1(1), 11-22.

Aulakh, P. S., Kotabe, M., \& Teegen, H. (2000). Export strategies and performance of firms from emerging economies: Evidence from Brazil, Chile, and Mexico. Academy of Management Journal, 43(3), 342-361. doi: $10.5465 / 1556399$

Baldauf, A., Cravens, D. W., \& Wagner, U. (2000). Examining determinants of export performance in small open economies. Journal of World Business, 35(1), 61-79.

Boehe, D. M., \& Cruz, L. B. (2010). Corporate social responsibility, product differentiation strategy and export performance. Journal of Business ethics, 91(2), 325-346.

Brouthers, L. E., \& Xu, K. (2002). Product stereotypes, strategy and performance satisfaction: The case of Chinese exporters. Journal of International Business Studies, 33(4), 657-677.

Chen, J., Sousa, C. M., \& He, X. (2016). The determinants of export performance: a review of the literature 2006-2014. International Marketing Review, 33(5), 626-670. 
Chugan, P. K., \& Singh, S. (2014). Taxonomy for measurement of firm-level export performance. Universal Journal of Industrial and Business Management 2(1), 6-12.

Eng, L. G. (1994). Using generic strategies: Some caveats. Singapore Management Review, 16(1), 43-48.

Erdil, T. S., \& Özdemir, O. (2016). The determinants of relationship between marketing mix strategy and drivers of export performance in foreign markets: An application on Turkish clothing industry. Procedia-Social and Behavioral Sciences, 235, 546-556.

Hedges, L. V., \& Olkin, I. (1980). Vote-counting methods in research synthesis. Psychological Bulletin, 88(2), 359-369.

Hughes, M., Martin, S. L., Morgan, R. E., \& Robson, M. J. (2010). Realizing product-market advantage in high-technology international new ventures: The mediating role of ambidextrous innovation. Journal of International Marketing 18(4), 1-21.

Katsikeas, C. S., Leonidou, L. C., \& Morgan, N. A. (2000). Firm-level export performance assessment: review, evaluation, and development. Journal of the Academy of Marketing Science, 28(4), 493-511.

Kumlu, Ö. (2014). The effect of intangible resources and competitive strategies on the export performance of small and medium-sized enterprises. Procedia-Social and Behavioral Sciences, 150, 24-34.

Leonidou, L. C., Fotiadis, T. A., Christodoulides, P., Spyropoulou, S., \& Katsikeas, C. S. (2015). Environmentally friendly export business strategy: Its determinants and effects on competitive advantage and performance. International Business Review, 24(5), 798-811.

Leonidou, L. C., Katsikeas, C. S., \& Coudounaris, D. N. (2010). Five decades of business research into exporting: A bibliographic analysis. Journal of International Management, 16(1), 78-91.

Leonidou, L. C., Palihawadana, D., \& Theodosiou, M. (2011). National exportpromotion programs as drivers of organizational resources and capabilities: effects on strategy, competitive advantage, and performance. Journal of International Marketing, 19(2), 1-29.

Ling-yee, L., \& Ogunmokun, G. O. (2001). Effect of export financing resources and supply-chain skills on export competitive advantages: implications for superior export performance. Journal of World Business, 36(3), 260279. 
126 | AN INVESTIGATION OF THE IMPACT OF COMPETITIVE STRATEGY ON EXPORT PERFORMANCE: A LITERATURE REVIEW IN THE PERIOD 1998 - 2018

Man, M. M. K. (2009). The Relationship between Distinctive Capabilities, Strategy Types, Environment and the Export Performance of Small and Medium-Sized Enterprises of the Malaysian Manufacturing Sector. Management (18544223), 4(3).

Martin, S. L., Javalgi, R. G., \& Cavusgil, E. (2017). Marketing capabilities, positional advantage, and performance of born global firms: Contingent effect of ambidextrous innovation. International business review, 26(3), 527-543.

Miller, D., \& Friesen, P. H. (1986). Porter's (1980) generic strategies and performance: an empirical examination with American data: part I: testing Porter. Organization Studies, 7(1), 37-55.

Mintzberg H. (1988). Generic strategies: toward a comprehensive framework. Advances in strategic management 5(1), 1-67.

Morgan, N. A., Katsikeas, C. S., \& Vorhies, D. W. (2012). Export marketing strategy implementation, export marketing capabilities, and export venture performance. Journal of the Academy of Marketing Science, 40(2), 271-289.

Murray, A. I. (1988). A contingency view of Porter's "generic strategies". Academy of management review, 13(3), 390-400.

Murray, J. Y., Gao, G. Y., \& Kotabe, M. (2011). Market orientation and performance of export ventures: the process through marketing capabilities and competitive advantages. Journal of the Academy of Marketing Science, 39(2), 252-269.

Njegić, K., \& Đokić, I. (2018). Antecedents of price adaptation strategy and its impact on export performance of Serbian firms. Škola biznisa, 1/2018, 120.

Okpara, J. O. (2012). An exploratory study of international strategic choices for exporting firms in Nigeria. Thunderbird International Business Review, 54(4), 479-491.

Oliveira, J. S., Cadogan, J. W., \& Souchon, A. (2012). Level of analysis in export performance research. International Marketing Review, 29(1), 114-127.

Papadopoulos, N., \& Martín, O. M. (2010). Toward a model of the relationship between internationalization and export performance. International Business Review, 19(4), 388-406.

Porter M. E. (1979). How competitive forces shape strategy. Harvard Business Review 57(2), 137-145. 
Porter M. E. (1985). Competitive advantage: creating and sustaining superior performance. New York: FreePress.

Porter, M. E. (1980). Industry structure and competitive strategy: Keys to profitability. Financial analysts journal, 36(4), 30-41.

Porter, M. E. (1981). The contributions of industrial organization to strategic management. Academy of management review, 6(4), 609-620.

Radosavljević, K. (2017). Uticaj marketinški orijentisane strategije na izvozne performanse predeuzeća. Beograd: Geoekonomski fakultet, Megatrend univerzitet.

Rua, O., França, A., \& Fernández Ortiz, R. (2018). Key drivers of SMEs export performance: the mediating effect of competitive advantage. Journal of Knowledge Management, 22(2), 257-279.

Salavou, H. E., \& Halikias, J. (2009). Strategy types of exporting firms: a view on the basis of competitive advantage. European Business Review, 21(2), 144-158.

Shepherd W. G. (1979). The economics of industrial organization. Englewood Cliffs, N.J. Prentice-Hall.

Shoham, A. (1998). Export performance: A conceptualization and empirical assessment. Journal of international marketing, 6(3), 59-81.

Slater, S. F., \& Olson, E. M. (2001). Marketing's contribution to the implementation of business strategy: An empirical analysis. Strategic Management Journal, 22(11), 1055-1067.

Sousa, C. M., Martínez-López, F. J., \& Coelho, F. (2008). The determinants of export performance: A review of the research in the literature between 1998 and 2005. International Journal of Management Reviews, 10(4), 343-374.

Theodosiou, M., \& Leonidou, L. C. (2003). Standardization versus adaptation of international marketing strategy: an integrative assessment of the empirical research. International business review, 12(2), 141-171.

Woodruff, C., \& Zenteno, R. (2007). Migration networks and microenterprises in Mexico. Journal of development economics, 82(2), 509-528.

Zou, S., \& Stan, S. (1998). The determinants of export performance: a review of the empirical literature between 1987 and 1997. International marketing review, 15(5), 333-356. 
Zou, S., Fang, E., \& Zhao, S. (2003). The effect of export marketing capabilities on export performance: an investigation of Chinese exporters. Journal of International Marketing, 11(4), 32-55.

\section{APPENDIX}

EP measurements used in the reviewed studies

\begin{tabular}{|c|c|}
\hline Author(s) & EP measurement \\
\hline $\begin{array}{l}\text { Aulakh et al. } \\
\qquad(2000)\end{array}$ & $\begin{array}{c}\text {-composite measure of EP: overall contribution of exports to sales growth, } \\
\text { market share growth, improvement of competitive position, export } \\
\text { profitability growth }\end{array}$ \\
\hline $\begin{array}{l}\text { Baldauf et al. } \\
\quad(2000)\end{array}$ & $\begin{array}{l}\text {-export effectiveness (composite measure of the contribution of export to } \\
\text { the growth of export sales volume, export intensity, market share, and the } \\
\text { number of export markets) } \\
\text {-export intensity } \\
\text { - export sales (revenue) }\end{array}$ \\
\hline $\begin{array}{l}\text { Ling-Yee, \& } \\
\text { Ogunmokun } \\
\quad(2001) \\
\end{array}$ & $\begin{array}{l}\text {-financial EP: export sales volume, export sales growth } \\
\text {-strategic EP: the achievement of export strategic goals }\end{array}$ \\
\hline $\begin{array}{l}\text { Brouthers, \& Xu } \\
(\text { (2002) }\end{array}$ & $\begin{array}{l}\text {-satisfaction with EP (satisfaction with export sales growth, export } \\
\text { profitability, overall EP, export market share) }\end{array}$ \\
\hline Zou et al. (2003) & $\begin{array}{l}\text {-financial EP (subjective assessment of the satisfaction with export } \\
\text { profitability, export sales, export sales growth) }\end{array}$ \\
\hline Man (2009) & -export sales revenue \\
\hline $\begin{array}{c}\text { Salavou, \& } \\
\text { Halikias (2009) }\end{array}$ & -export profitability \\
\hline $\begin{array}{l}\text { Boehe, \& Cruz } \\
\quad(2010)\end{array}$ & $\begin{array}{l}\text {-composite EP (subjective assessment of: export sales volume, export } \\
\text { sales revenue, export profitability, export market share, overall EP) }\end{array}$ \\
\hline $\begin{array}{l}\text { Hughes et al. } \\
\quad(2010)\end{array}$ & $\begin{array}{c}\text {-composite EP (subjective assessment of effectiveness, efficiency, and } \\
\text { adaptiveness of export activities) }\end{array}$ \\
\hline $\begin{array}{l}\text { Leonidou } \text { et al. } \\
\quad \text { (2011) }\end{array}$ & $\begin{array}{c}\text {-financial EP (subjective assessment of export sales volume, export } \\
\text { market share, export profitability, and export intensity) } \\
\text {-export market performance (the level in which a firm provided value to } \\
\text { export customers, retained valued export customers, acquired new export } \\
\text { customers, and the assessment of firms' reputation among export } \\
\text { customers) }\end{array}$ \\
\hline $\begin{array}{l}\text { Murray et al. } \\
\quad(2011)\end{array}$ & $\begin{array}{l}\text {-financial EP (export profits, export sales, export sales growth) } \\
\text {-strategic EP: the achievement of export strategic goals } \\
\text {-product performance (number of successful new products, speed of } \\
\text { getting new products to the market, and product innovations in } \\
\text { comparison with three major competitors) }\end{array}$ \\
\hline Okpara (2012) & $\begin{array}{l}\text {-export sales growth } \\
\text {-export profitability } \\
\text {-overall EP }\end{array}$ \\
\hline
\end{tabular}




\section{Author(s) $\quad$ EP measurement}

Kumlu (2014) -composite EP (satisfaction with export sales growth, export sales profit, and export sales volume and profit; contribution of exports to total turnover growth; the level of foreign customers satisfaction)

\begin{tabular}{|c|c|}
\hline $\begin{array}{l}\text { Leonidou et al. } \\
\quad(2015)\end{array}$ & $\begin{array}{l}\text {-export market performance (rate of acquiring foreign customers; rate of } \\
\text { maintaining foreign customers, rate of sales increase by current foreign } \\
\text { customers; foreign customer satisfaction; foreign customer loyalty; } \\
\text { reputation of company among foreign buyers) } \\
\text {-financial EP (export profits; export sales; export sales intensity; return on } \\
\text { export sales; return on export-related investment; return on export-related } \\
\text { capital) }\end{array}$ \\
\hline $\begin{array}{l}\text { Erdil, \& Özdemir } \\
\text { (2016) }\end{array}$ & $\begin{array}{c}\text {-financial EP (export revenue/number of employees; export intensity; } \\
\text { export intensity growth) }\end{array}$ \\
\hline $\begin{array}{l}\text { Martin et al. } \\
\quad(2017)\end{array}$ & $\begin{array}{l}\text {-composite measure of EP: (effectiveness: the extent to which } \\
\text { organizational goals and objectives are met; efficiency: the relationship } \\
\text { between performance financial outcomes and the inputs required to } \\
\text { achieve them; adaptiveness: the operational ability to respond to } \\
\text { environmental changes) }\end{array}$ \\
\hline Rua e & $\begin{array}{l}\text {-composite measure of EP: subjective assessment of export profitability, } \\
\text { export sales volume, growth in export activities in the last three years, the } \\
\text { expansion of export operations in the last three years, overall EP }\end{array}$ \\
\hline
\end{tabular}

Note. Authors' research.

Delivered: 09.04.2019.

Accepted: 05.12.2019. 